\title{
CONTAR PARA COMPRENDER: CIERRE DE ESCUELAS RURALES MUNICIPALES EN CHILE Y SUS IMPLICANCIAS PARA LAS COMUNIDADES
}

\author{
CARMEN NúÑEZ MUÑoZO ${ }^{1}$ \\ Bryan GonzÁlez NiCULCAR ${ }^{2}$ \\ Paula Ascorra Costa ${ }^{3}$ \\ SHAUN GRECH ${ }^{4}$
}

\begin{abstract}
RESUMEN: Frente a los sucesivos cierres de escuelas rurales, se presenta un estudio cuyo objetivo es conocer su real dimensión. Se presenta una caracterización estadística de las escuelas básicas rurales cerradas en Chile entre el 2000 y 2016. Se analizó el número de escuelas rurales en funcionamiento y cerradas a nivel nacional, regional, por dependencia administrativa, Índice de Vulnerabilidad Escolar y matrícula. Los resultados muestran que los cierres no son hechos aislados, sino una tendencia que alcanza dimensiones preocupantes. La media del IVE de las escuelas cerradas muestra que los cierres afectan principalmente a la población más pobre. Desde estos resultados se interroga la política educativa, su relación con el debilitamiento de la educación rural y sus consecuencias en las comunidades rurales.
\end{abstract}

Palabras-clave: Educación rural. Cierre de escuelas. Escuelas básicas. Estudio cuantitativo. Comunidad.

\section{COUNT TO UNDERSTAND: CLOSURE OF RURAL MUNICIPALS SCHOOLS IN CHILE AND THEIR IMPLICATIONS FOR COMMUNITIES}

\begin{abstract}
In response to the successive closures of rural schools, this paper reports on a study, the objective of which is to know the real magnitude of this phenomenon. The paper presents a statistical overview of elementary schools shut down in Chile between 2000 and 2016. We analyzed number of closed and operating rural schools at national and regional level, according to Administrative dependency, School Vulnerability index and enrolment. Results show that these closures are not isolated facts, but a trend that reaches troubling dimensions. The School Vulnerability index average also demonstrates how these closures mainly affect the poorest populations. Through these findings, we interrogate the educational policy, its linkages with the weakening of rural education, and its consequences for the rural communities.
\end{abstract}

Keywords: Rural education. School closing. Elementary schools. Quantitative study. Community.

\footnotetext{
1.Pontificia Universidad Católica de Valparaíso, Facultad de Filosofía y Educación, Escuela de Psicología - Valparaíso, Chile. E-mail: carmen.nunez@pucv.cl

2.Pontificia Universidad Católica de Valparaíso, Facultad de Filosofía y Educación, Escuela de Psicología - Valparaíso, Chile. E-mail: bryan.gonzalez.n@mail.pucv.cl

3.Pontificia Universidad Católica de Valparaíso, Facultad de Filosofía y Educación, Escuela de Psicología - Valparaíso, Chile. E-mail: paula.ascorra@pucv.cl

4.The Critical Institute, Centre for Global Disability Research - Valeta, Malta. E-mail: s.grech@criticalinstitute.org

Realizado en el marco del proyecto FONDECYT No 1161338
} 


\section{CONTAR PARA ENTENDER: FECHAMENTO DAS ESCOLAS MUNICIPAIS RURAIS NO CHILE E SUAS IMPLICAÇÕES PARA AS COMUNIDADES}

RESUMO: Em resposta ao fechamento sucessivo de escolas rurais, este artigo relata um estudo, cujo objetivo é conhecer a real magnitude desse fenômeno. Este artigo apresenta uma caracterização estatística das escolas básicas rurais fechadas no Chile entre 2000 e 2016. Se você analisar o número de escolas rurais em nível de funcionamento e nível nacional, regional, por dependência administrativa, Índice de Vulnerabilidade Escolar e Matrícula. Os resultados mostram que esses fechamentos não são fatos isolados, mas uma tendência que atinge dimensões preocupantes. A média do índice de vulnerabilidade escolar também demonstra como esses fechamentos afetam principalmente as populações mais pobres. Através dessas descobertas, interrogamos a política educacional, seus vínculos com o enfraquecimento da educação rural e suas conseqüências para as comunidades rurais.

Palabras-chave: Educação rural. Fechamento da escola. Escola primária. Estudo quantitativo. Comunidade.

\section{Introducción}

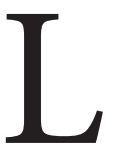

as políticas estatales en Chile han sido diseñadas sobre la consideración de un país urbano, en desmedro de un importante sector de la población que habita en zonas rurales. Según el Instituto Nacional de Estadísticas, el porcentaje de la población rural en Chile es $12.2 \%$ (INSTITUTO NACIONAL DE ESTADÍSTICAS, 2018). Sin embargo, un estudio reciente de la OCDE, con base en sus estándares, da cuenta que dicha población es de un 34.6 \% (OCDE, 2014). La disparidad en estos datos evidencia la falta de una definición de lo rural.

Lo anterior ha tenido altas implicancias para el desarrollo de las sociedades rurales, dando cuenta de la necesidad de implementar políticas públicas integrales que vayan más allá de lo económico y que reconozcan sus procesos de transformación social, cultural y tecnológica de las últimas décadas (RODRÍGUEZ, 2014). Ello implica avanzar en definiciones que incluyan la diversidad de ruralidades y aquellas dimensiones fundamentales para el desarrollo de la vida de los sujetos, entre las cuales destaca la educación.

No obstante, en el ámbito de la educación rural, y junto a una profunda lógica de educación de mercado, se observa una evidente desatención a las escuelas rurales y un progresivo debilitamiento, que se relaciona estrechamente con su tendencia al cierre. Nuestras investigaciones cualitativas dan cuenta que la escuela rural representa un motor de desarrollo comunitario, puesto que es el gran eje articulador de la cohesión social; su cierre por el contrario, se constituye en una amenaza que anticipa el despoblamiento y la muerte social de la comunidad (NÚÑEZ; SOTO, 2014; SOLÍS; NÚÑEZ, 2014). A pesar de lo anterior, las autoridades tienden a reducir la escuela a un servicio educativo dirigido a los estudiantes, desconociendo su valor comunitario y simbólico.

Es preciso señalar que no existen estadísticas oficiales sistemáticas de escuelas rurales cerradas, por lo que este estudio ${ }^{1}$ se propone conocer las reales dimensiones del fenómeno desde una perspectiva cuantitativa, de manera complementaria a los estudios cualitativos anteriores, buscando construir una mirada profunda y comprehensiva de la actual situación de escuelas rurales en Chile. 
El foco del análisis está puesto sobre las escuelas básicas municipales, puesto que constituyen el mayor número de establecimientos rurales a lo largo de todo el territorio; en el año 2016 existían un total de solo 213 establecimientos rurales de educación media frente a los más de 2.000 de educación básica (MINEDUC, 2017). El gran número de escuelas básicas responde a una política de Estado, que desde sus orígenes se propuso la expansión de la educación primaria rural para alfabetizar a los campesinos, lo que durante el gobierno de Frei-Montalva (1964-1970) se concretizó con el "Plan Extraordinario de Construcciones Escolares" (PÉREZ, 2018, p. 20). A su vez, junto a las escuelas municipales, existen en Chile otras de dependencia particular, las que se dividen en subvencionadas y privadas, las que son menores en número en la educación rural; si bien éstas no constituyen el foco, su comportamiento también es analizado con relación a las escuelas municipales, aunque de manera marginal.

\section{¿Qué es lo rural en Chile?}

En Chile lo rural es definido a partir de la dicotomía urbano-rural, donde lo rural es aquello que no es urbano (OCDE, 2014). Desde el Estado se entiende como "Entidad Urbana" a un conjunto de viviendas concentradas, con más de 2.000 habitantes, o entre 1.001 y 2.000, con el $50 \%$ o más de su población económicamente activa dedicada a actividades secundarias y/o terciarias. Excepcionalmente, los centros de turismo con más de 250 viviendas concentradas y que no alcanzan el requisito de población, se consideran entidades urbanas (INSTITUTO NACIONAL DE ESTADÍSTICAS, 2018). En consecuencia, lo rural corresponde a aquellas áreas con menos de 2.000 habitantes en que predominan las actividades primarias. De acuerdo a los datos del INE la población rural ha disminuido en el tiempo, si consideramos que en 1992 la población rural era de un 16,5 \% mientras que en 2002 correspondía a un $13,14 \%$ y en 2018 de 12,2\%. No obstante, en términos de número de personas que viven en las áreas rurales, es preciso referir que entre los años 2002 y 2017 se observa un aumento de 2.026 .322 a 2.149.740 (INSTITUTO NACIONAL DE ESTADÍSTICAS, 2002, 2018).

Por su parte, la OCDE establece que las unidades locales (como las municipalidades) se definen como rurales si su densidad de población es inferior a los 150 habitantes por kilómetro cuadrado; luego las regiones se clasifican en tres categorías:

- Región predominantemente rural (PR): más del 50 \% de la población vive en municipios rurales.

- Región intermedia (RI): entre un $15 \%$ y un 50 \% de la población vive en unidades locales rurales.

- Región predominantemente urbana (PU): menos del 15 \% de la población vive en unidades locales rurales.

Según esta categorización, la población rural en Chile es de un 34.6 \% (OCDE, 2014). La disparidad en los datos de INE y OCDE es significativa, lo que evidencia que distintas definiciones de lo rural son posibles, más allá de la clasificación tradicional en que Chile ha estado basando sus políticas públicas.

A su vez, los instrumentos de ordenamiento y planificación territorial tienen un alto énfasis en el desarrollo urbano, desatendiendo las áreas rurales (DATTWYLER et al., 2016; JIMÉNEZ et al., 2018). Esto se replica en distintos países de la región, lo que a su vez ha impulsado pensar la ruralidad no sólo desde una perspectiva cuantitativa, sino que desde la comprensión de sus particularidades; declarando así la existencia de una nueva ruralidad (KAY, 2009; RODRÍGUEZ; SALAS, 2010; ALBARRÁN, 2016). La nueva ruralidad permite comprender las transformaciones de lo rural, y es una alternativa 
conceptual frente a aquellos análisis que plantean la desaparición de lo rural frente a la expansión de lo urbano. Efectivamente los territorios rurales han atravesado grandes cambios, y si antaño se caracterizaban por el desarrollo de actividades principalmente agrarias, hoy éstas han disminuido notoriamente llevando a lo que se ha denominado como "desagrarización" del campo y transitando hacia una pluriactividad, donde coexisten actividades agroindustriales, recreación y ecoturismo (RODRÍGUEZ; SALAS, 2010; ALBARRÁN, 2016). Al mismo tiempo, las fronteras con lo urbano se han complejizado y se han vuelto más difusas, y a la migración desde el campo a la ciudad que caracterizó la segunda mitad del siglo XX, se le ha sumado la migración desde la ciudad al campo en los últimos años (TRIMANO, 2015). Con el concepto de nueva ruralidad -o nuevas ruralidades (RODRÍGUEZ; SALAS, 2010)- se busca superar la dicotomía rural/urbano, así como dar relevancia a la diversidad de relaciones sociales que ocurren en el territorio, buscando poner el foco en los recursos de las comunidades rurales y no solo en sus carencias (ROJAS, 2017).

Si aceptamos que la definición actual de las áreas rurales en Chile invisibiliza la complejidad del territorio y que, de acuerdo a estándares internacionales, un alto porcentaje de los ciudadanos vive en áreas rurales, resulta perentorio contar con políticas integrales (OCDE, 2014) que visibilicen su existencia y que garanticen la mejora de su calidad de vida. En este sentido, la educación en las áreas rurales cumple un rol fundamental a nivel individual, comunitario y local.

\section{Cierre y Consolidación de escuelas rurales como política de Estado}

Si bien en Chile el cierre de las escuelas municipales depende de cada municipio, existen elementos que hacen pensar que éste es un fenómeno nacional. En algunos países el cierre se ha articulado como una política de Estado, principalmente para optimizar recursos públicos. Entre 1990 y el 2010 Finlandia cerró 2.117 escuelas denominadas como “pequeñas y comprehensivas", las que representan el $65 \%$ del total (AUTTI; HYRY-BEIHAMMER, 2014). En España existió una política nacional de concentración a fines de los '70, en que se cerraron una gran cantidad de escuelas rurales (OLIVARES et al., 2015). En China, se diseñó una política de consolidación de escuelas rurales oficialmente declarada, en los inicios de la década de los 80' y en el 2001; de este modo, el año 2004 el Ministerio de Educación declaró haber cerrado un total de 40.000 escuelas básicas y medias rurales (MEI; FANG; YUANYAN, 2013).

En el contexto latinoamericano, en Argentina, entre 1977 y 1983 se realizó una concentración de escuelas rurales, cerrándose cerca de 195 (RODRÍGUEZ, 2008). En Uruguay, entre 1994 y 1999, se aplicó una política de reestructuración de la educación rural, generando nucleamientos o consolidaciones por medio de cierres de escuelas rurales con baja matrícula (MARTINIS; REDONDO, 2006). En Chile, si bien no ha sido explicitada como una política propiamente tal, sí han existido iniciativas desde el nivel central que lo han planteado como una solución para el déficit municipal generado por la baja en la matrícula y como una posibilidad de mejora de los procesos de socialización de los estudiantes (GOBIERNO DE CHILE, 2010).

Sea mediante una política estatal explícita o implícita, el cierre de escuelas ha sido argumentado como una medida de optimización de recursos públicos. Es así como la racionalidad económica, entendida como la maximización en la relación costos y beneficios (BORÓN, 2003) es la que se impone como lógica imperante en la toma de decisiones.

\section{Los argumentos frente al cierre y la competencia por la matrícula}

Durante la dictadura en Chile entre 1973 y 1990, se realizaron cambios constitucionales que introdujeron medidas neoliberales en el sistema educativo, que fueron pioneras en el mundo. Así, en la década de los ' 80 se establece: 
[...] la consolidación del sistema de subvenciones educacionales, por medio del cual se financia la educación privada y municipal, y la descentralización de la función de gestión del Estado, con el traspaso a los municipios de todos los establecimientos educacionales que dependían del gobierno central. Con esto se estableció un sistema en el que el Ministerio de Educación paga una subvención mensual por alumno que asiste a clases en las escuelas, sean estas municipales o particulares, a las que los padres eligen enviar a sus hijos. (MARCEL; TOKMAN, 2005, p.11).

Con lo anterior se establece un sistema de voucher en lugar de un aporte basal a las escuelas (ARVESETH, 2014) y se crean 3 tipos, según su dependencia financiera: particulares privadas, municipales y particulares-subvencionadas. De éstas, las 2 últimas reciben subvención del Estado. Así, el voucher y la creación de las escuelas particulares-subvencionadas introducen una lógica de competencia por la matrícula, especialmente entre éstas y las municipales, puesto que las privadas siempre han tenido una matrícula sectorizada que no representa más del 7 \% (MINEDUC, 2017).

Dentro de los argumentos para el cierre, aquel que se plantea con mayor énfasis en los instrumentos municipales ( $\mathrm{PADEM}^{2}$ ), es la baja matrícula de las escuelas. Para corregir dicha situación, en el caso de las escuelas rurales municipales, se creó en 1998 el "piso rural", cuyo objetivo es financiar establecimientos rurales con matrícula no superior a 17 alumnos y con severo aislamiento geográfico (DARVILLE; RODRÍGUEZ, 2007). Sin embargo, de 757 establecimientos que originalmente cumplían los requisitos para obtener el beneficio, se disminuyó a 521 en el año 2004³ y en años posteriores, se publican decretos que privan a ciertos establecimientos con dos variables:

- $\quad$ El IVE-SINAE de estas en su último año de funcionamiento.

- El número de matrícula que la escuela tenía al año anterior a su cierre.

Por último, con el fin de ampliar la rurales de acceder a esta subvención. Debido al financiamiento vía voucher mencionado anteriormente, la baja matrícula de las escuelas rurales hace que el municipio reciba menos subvención por este concepto. De este modo, el argumento económico posiciona a la escuela como un gasto y no como una inversión necesaria para los sectores rurales.

Un segundo argumento, alude a la calidad de la educación (GOBIERNO DE CHILE, 2010), considerando como indicador los puntajes de la prueba estandarizada SIMCE ${ }^{4}$ de estas escuelas, los que se encontrarían por debajo el promedio nacional. En los últimos años ha existido un fuerte debate académico y ciudadano ${ }^{5}$ respecto al peso que ha adquirido el SIMCE en la educación, puesto que la Ley General de Educación (LGE) establece que una escuela con bajos resultados pierde su reconocimiento ministerial si no mejora en 4 años, pudiendo ser cerrada (CHILE, 2009). Los datos muestran que los resultados de la prueba se correlacionan fuertemente con el nivel socioeconómico (NSE) de los estudiantes y el nivel de escolaridad de sus padres, generándose brechas importantes con estudiantes de mayor NSE y escolaridad parental (AGENCIA DE LA CALIDAD DE LA EDUCACIÓN, 2015). Son las escuelas que reciben a estudiantes de menor NSE las que corren mayor riesgo de cierre, puesto que sus puntajes son más bajos. Este argumento de la calidad ha sido planteado en mayor medida por las autoridades ministeriales, mientras que es aplicado de manera menos sistemática por las autoridades municipales.

Un tercer argumento para el cierre señala las pocas posibilidades de socialización que entregan estas escuelas a los niños, por ser pequeñas (GOBIERNO DE CHILE, 2010). Sin embargo, esta afirmación no ha sido respaldada por estudios nacionales o internacionales que den cuenta de ello. 


\section{Cambio de denominación}

Un fenómeno muy reciente lo constituye el cambio en la denominación oficial de muchas escuelas rurales, que pasan a ser consideradas urbanas. Esto se debe a que dejan de cumplir con la definición que dispone el Artículo 12 del Decreto con Fuerza de Ley de Educación № 2, donde se entenderá como escuela rural a aquel establecimiento:

[...] que se encuentre ubicado a más de cinco kilómetros del límite urbano más cercano, salvo que existan accidentes topográficos importantes u otras circunstancias permanentes derivadas del ejercicio de derechos de terceros que impidan el paso y obliguen a un rodeo superior a esta distancia o que esté ubicado en zonas de características geográficas especiales. (Art. 12 DFL de Educación No2, 1998).

Dada esta definición, en la que el aspecto geográfico es el único determinante, los cambios en los planes reguladores de las localidades -como la pavimentación de los caminos que antes eran de tierra-, repercuten en la denominación de las escuelas. El cambio afecta de manera directa, pues la escuela pierde beneficios y subvenciones importantes para su funcionamiento.

\section{Metodología}

Se realizó un análisis descriptivo longitudinal del comportamiento de las escuelas básicas rurales entre los años 2000 y 2016 a nivel nacional y regional ${ }^{6}$, con el fin de identificar el número de escuelas cerradas. Además, se vincularon las escuelas rurales municipales cerradas en el país entre los años 2010 y 2016 con su Índice de Vulnerabilidad Escolar (IVE-SINAE ${ }^{7}$ ) y número de matrícula al año de cierre.

Para el análisis se trabajó con la población total y se utilizaron dos fuentes officiales ${ }^{8}$ los Directorios de establecimientos educacionales que dispone el Ministerio de Educación (MINEDUC) del año 2000 a 2016 y las bases de datos de Prioridades IVE-SINAE elaboradas por la Junta Nacional de Auxilio Escolar y Becas (JUNAEB) del año 2010 a 2016.

El procedimiento para el análisis de cierre consistió en una revisión año a año y escuela por escuela, es decir, se observó el comportamiento de cada una de éstas durante el periodo referido utilizando su Rol en la Base de Datos $\left(\mathrm{RBD}^{9}\right)$. Con el propósito de obtener un número preciso de cierre, se diferenciaron en el análisis cuatro estados de las escuelas:

- Cambia a urbana: cambio de denominación de rural pasa a ser urbana

- Cierra: cese de sus funciones, persistiendo en este estado hasta el último año de análisis.

- En funcionamiento: abierta durante el año escolar 2016.

- En receso: establecimientos que, mediante su sostenedor, han solicitado su receso temporal.

En segundo lugar, se realizó un análisis que vinculó las escuelas rurales que cerraron durante el periodo 2010 a $2016^{10}$ comprensión del comportamiento de los establecimientos ubicados en sectores rurales, se realizó un análisis por dependencia, donde se evidencia el número de escuelas básicas rurales particulares-subvencionadas y particulares-privadas a nivel regional y nacional en funcionamiento entre el año 2000 y 2016. 
Tabla 1. Comportamiento escuelas rurales municipales entre los años 2000 y 2016.

\begin{tabular}{ccccc}
\hline Región & $\begin{array}{c}\text { Cambia a } \\
\text { Urbana }\end{array}$ & Cierra & \multicolumn{2}{c}{ Estado } \\
\cline { 4 - 5 } & & & En funcionamiento 2016 & En receso 2016 \\
\hline Arica y Parinacota -Tarapacá & 3 & 3 & 16 & 0 \\
\hline Antofagasta & 1 & 2 & 37 & 1 \\
\hline Atacama & 0 & 9 & 272 & 0 \\
\hline Coquimbo & 23 & 47 & 131 & 2 \\
\hline Valparaíso & 49 & 27 & 245 & 0 \\
\hline O’Higgins & 8 & 78 & 388 & 4 \\
\hline Maule & 4 & 106 & 506 & 1 \\
\hline Biobío & 3 & 217 & 313 & 18 \\
\hline La Araucanía & 0 & 199 & 630 & 0 \\
\hline Los Ríos y Los Lagos & 5 & 269 & 29 & 0 \\
\hline Aysén & 1 & 5 & 16 & 0 \\
\hline Magallanes & 1 & 3 & 111 & 39 \\
\hline RM & 0 & 12 & 2765 & \\
\hline Total & 98 & 977 & & 2 \\
\hline
\end{tabular}

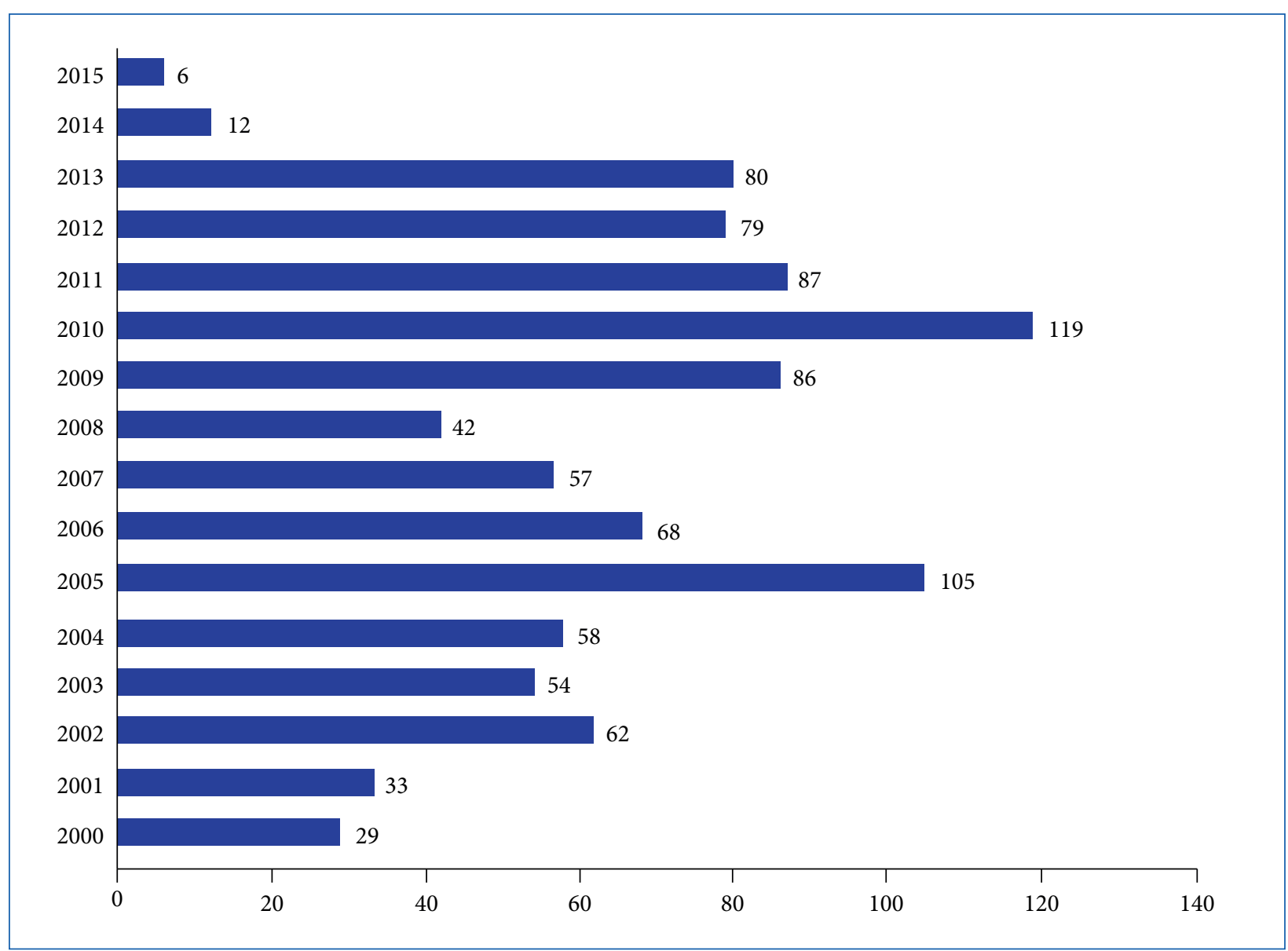

Figura 1. Numero de escuelas rurales municipales cerradas a nivel nacional entre los años 2000 - 2016y. 


\section{Resultados}

Entre los años 2000 y 2016 se cerraron en Chile 977 (Tabla 1) escuelas básicas rurales municipales, de un total de 3.807 que existían en el año 2000. En promedio se cierran 61 escuelas por año; no obstante, se pueden observar dos peak de cierre, uno en el año 2005 con 105 escuelas rurales municipales cerradas y el otro el 2010 con 119 (Fig. 1).

Exceptuando los años 2005 y 2010, es posible observar que si bien el número de escuelas que cierran varía año a año, queda demostrado que cada año se cierran al menos 6 escuelas. Además, existe una tendencia al aumento del cierre de escuelas desde el año 2000 hasta el año 2013 (Fig. 1). No obstante, en los años 2014 y 2015 se expresa una disminución en el número de cierre con 12 y 6 escuelas cerradas en el país respectivamente.

En el año 2016 existían un total de 39 escuelas rurales municipales en receso (Tabla 1), 19 de ellas desde el 2015 y 17 desde el 2016; siendo las regiones de Los Ríos y Los Lagos las que concentran un mayor número de escuelas bajo esta categoría (46,1 \%), seguidas de la región de La Araucanía (20,5 \%). Conviene señalar que las escuelas en receso no se comportan uniformemente, algunas vuelven a funcionar en algún periodo y otras no. El periodo de tiempo que se mantienen en receso por lo general es de uno o dos años.

El análisis regional muestra que la zona centro y sur concentran mayor número de cierres: región del Maule (106), Biobío (217), La Araucanía (199), de Los Ríos y Los Lagos (269); concentrando un $80,9 \%$ del total de las escuelas cerradas a nivel nacional (Tabla 1), las que a su vez concentran el mayor número de escuelas rurales. Sin embargo, si consideramos las escuelas que se encontraban en funcionamiento en estas regiones en el año 2000 (Tabla 2) se observa que en La Araucanía se han cerrado un 38,9 \% de las escuelas rurales, en la del Biobío un 30,2 \% y en Los Ríos y Los Lagos un 29,7 \%,

Tabla 2. Escuelas rurales municipales, particulares subvencionadas y privadas en funcionamiento.

\begin{tabular}{lccc}
\hline Año & Escuelas Municipales & Escuelas Part. Subvencionadas y Privadas & Total \\
\hline 2000 & 3807 & 804 & 4611 \\
\hline 2001 & 3773 & 833 & 4606 \\
\hline 2002 & 3728 & 848 & 4576 \\
\hline 2003 & 3666 & 872 & 4538 \\
\hline 2004 & 3618 & 862 & 4480 \\
\hline 2005 & 3570 & 870 & 4440 \\
\hline 2006 & 3459 & 893 & 4352 \\
\hline 2007 & 3395 & 891 & 4286 \\
\hline 2008 & 3336 & 889 & 4225 \\
\hline 2009 & 3298 & 896 & 4194 \\
\hline 2010 & 3217 & 893 & 4110 \\
\hline 2011 & 3098 & 872 & 3970 \\
\hline 2012 & 3012 & 851 & 3863 \\
\hline 2013 & 2932 & 819 & 3751 \\
\hline 2014 & 2814 & 797 & 3611 \\
\hline 2015 & 2778 & 790 & 3568 \\
\hline 2016 & 2765 & 774 & 3537 \\
\hline
\end{tabular}


mientras que en el Maule se habría cerrado un 21,2 \%. Es llamativo que en la región de O’Higgins, las 78 escuelas que han cerrado equivaldrían a un 24,2 \% de las escuelas en funcionamiento en el año 2000.

En cuanto a la zona norte, destaca la región de Coquimbo donde se han cerrado un total de 47, lo que en relación a las escuelas que se encontraban en funcionamiento en la región en el año 2000 corresponde a un 13,7 \%. Por su parte en las regiones de Valparaíso y Metropolitana, que corresponden al centro del país, se han cerrado en total 39 escuelas.

Es importante considerar las escuelas que se encuentran en funcionamiento (Tabla 2), ya que a nivel nacional estas han disminuido de manera progresiva a lo largo de los años. En el año 2000 existían 3.807 escuelas básicas rurales municipales en funcionamiento y 804 particulares subvencionadas/particulares privadas. En el año 2016 existe un comportamiento similar de tendencia a la disminución, donde las municipales disminuyen a 2.765 y las particulares subvencionadas y privadas a 774. Sin embargo, en el caso de las particulares subvencionadas y privadas la disminución no es año a año, ya que desde el año 2000 a 2009 su tendencia es a aumentar y desde el 2010 hasta el 2016 la tendencia es a disminuir. Es decir, durante 9 años hubo una tendencia al aumento de las escuelas particulares subvencionadas y privadas, mientras que las municipales fueron solo disminuyendo durante todo el periodo analizado.

En lo que refiere al análisis regional, los resultados muestran que en las regiones del extremo norte y extremo sur (Arica y Parinacota, Antofagasta, Magallanes y Aysén), si bien se registran cierres, también se evidencian aperturas de establecimientos municipales, mientras que en el resto de las regiones se observa una disminución progresiva de las escuelas. En este caso la región de Antofagasta es la única que registra un aumento de 3 escuelas rurales comparando las existentes en los años 2000 y 2016; sin embargo, es necesario precisar que este aumento de escuelas no anula los 3 cierres que se registran en la región.

Un aspecto interesante, es la categoría emergente de este análisis, que da cuenta de las escuelas rurales que, si bien no han cerrado, han cambiado de denominación de área (Tabla 1). En esta dimensión destacan la región de Coquimbo y la de Valparaíso.

\section{Índice de vulnerabilidad escolar (IVE) y escuelas básicas rurales municipales cerradas entre los años $2010-2016$}

El IVE corresponde al porcentaje de estudiantes definidos como vulnerables en un determinado establecimiento educativo. Para definir la vulnerabilidad individual se utiliza una metodología llamada SINAE:

[...] que identifica según prioridades de atención las desigualdades en que se encuentra la población escolar, con el fin de permitir la posterior entrega de los apoyos específicos que requieran para terminar con éxito sus 12 años de escolaridad (JUNAEB, 202011).

El instrumento establece tres prioridades: 1) población que se encuentra en riesgo de subsistencia o extrema pobreza incluyendo la pobreza rural; 2) población pobre que presenta, entre otros factores, riesgos de deserción escolar y bajo rendimiento, y 3) aquellos que sin contar con los factores de riesgos de la primera y segunda prioridad, son definidos como pobres socioeconómicamente.

Antes de revisar las características de la población estudiantil respecto del IVE- SINAE de las escuelas cerradas, es preciso señalar que entre los años 2010 y 2016 se cerraron un total de 466 escuelas rurales municipales; es decir, un $39 \%$ del total de las escuelas municipales cerradas de todo

1 Ver https://www.junaeb.cl/como-funciona-el-sinae 


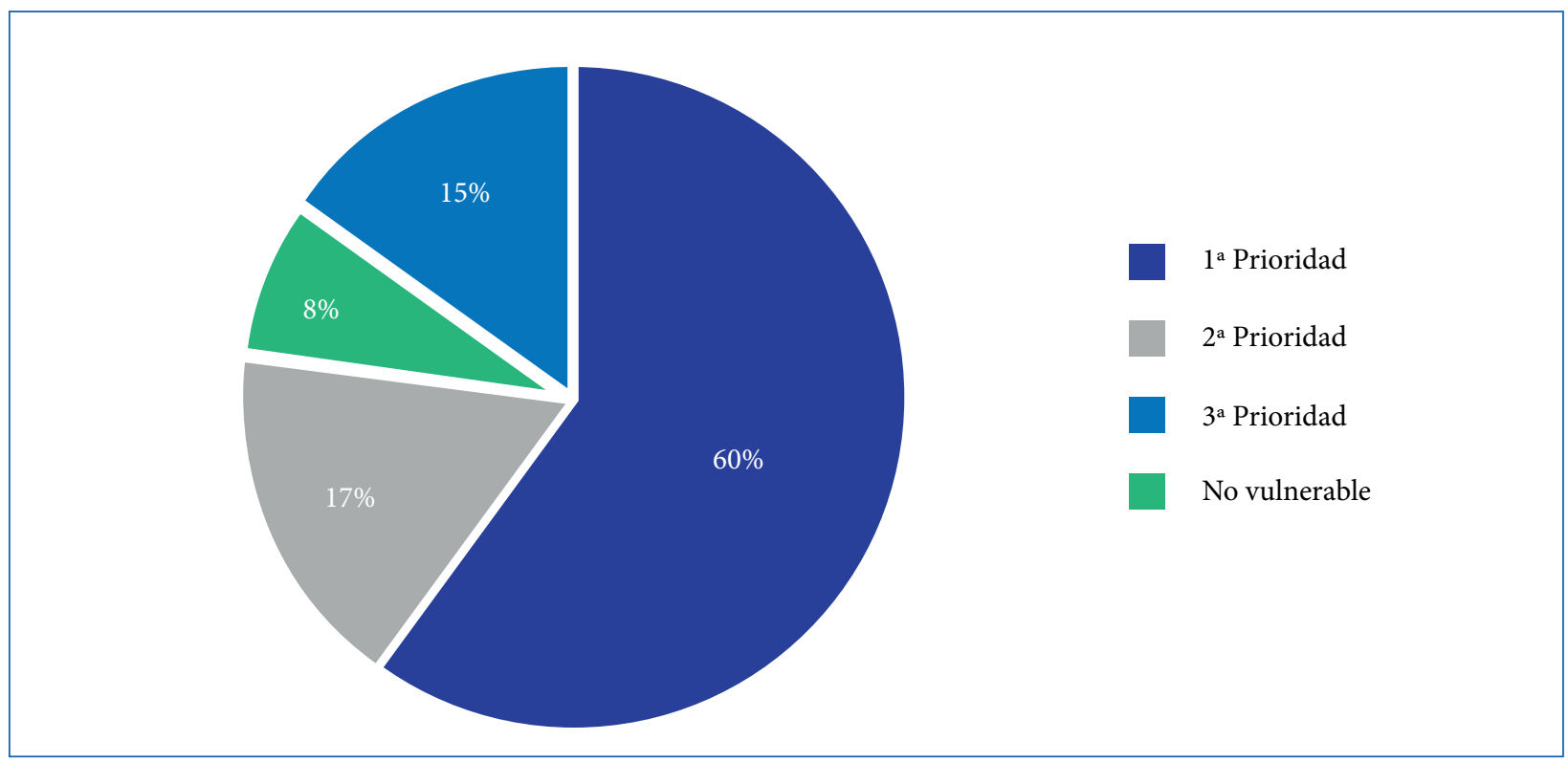

Figura 2. Prioridades IVE-SINAE escuelas rurales municipales cerradas entre los años 2010 - 2016.

el periodo analizado (2000-2016). En lo que refiere a este periodo, estas escuelas en su último año de funcionamiento tenían a nivel nacional un $60 \%$ de estudiantes vulnerables de primera prioridad, un $17 \%$ de segunda prioridad y un $15 \%$ de tercera prioridad (Fig. 2); conjunto que representa un 92 $\%$ de los estudiantes de dichas escuelas. Asimismo, es preciso mencionar que la mayor cantidad de escuelas cerradas tenían un IVE- SINAE cercano a $87,9 \%$ o superior a éste.

\section{Matrícula escuelas rurales municipales cerradas entre los años 2010 - 2016}

El análisis respecto de la matrícula indica que la mayor parte de las escuelas tienen un número de estudiantes menor que la media, es decir, que por lo general las escuelas cerradas tienen un número igual o inferior a 11 estudiantes. No obstante, existen algunos casos atípicos. En la región de Atacama se cerraron entre 2010 y 2016, tres escuelas rurales municipales con un promedio de 190 estudiantes; en la región de los Ríos se cerró un establecimiento con 202 estudiantes; en la región de La Araucanía se cerró una escuela que tenía una matrícula de 99 estudiantes, y en la región Metropolitana tres escuelas con un promedio de 142 estudiantes.

\section{Conclusiones}

Los análisis presentados son inéditos y no se encuentran sistematizados por parte de las autoridades ministeriales. Por primera vez es posible conocer la magnitud real del fenómeno de cierre de escuelas, el IVE de sus estudiantes y el cambio de denominación de rural a urbana. Estos son datos de suma relevancia para informar a quienes investigan y toman decisiones, del estado actual de la educación rural.

Si bien en Chile no existe una política pública declarada que comande el cierre de escuelas rurales como en otros países, los resultados evidencian su progresivo cierre. Esto muestra la magnitud del fenómeno en un periodo de tiempo acotado y que además, esto no corresponde a decisiones aisladas de cada municipio, sino a un fenómeno nacional. 
Las variaciones en el número de establecimientos rurales en funcionamiento entre los años 2000 y 2016 son significativamente menores en los particulares subvencionados y particulares pagados en comparación con las escuelas municipales. Esto refleja que el argumento de la baja en la tasa de natalidad detrás del cierre de escuelas es cuestionable, puesto que las únicas mayormente afectadas son las escuelas municipales; si el nacimiento de menos niños fuese la razón, todas las escuelas debieran verse afectadas por igual a lo largo del tiempo. Lo que se evidencia en la desigualdad de comportamiento entre escuelas de distinta dependencia, es el efecto de la competencia entre escuelas por la matrícula, que tiene una repercusión económica directa en la sustentabilidad de las escuelas, al basarse su financiamiento en el sistema de voucher. La competencia entre escuelas públicas y particulares subvencionadas ha caracterizado el panorama nacional en las últimas décadas. En el año 1981, el 78 \% de la matrícula se concentraba en escuelas municipales y en el año 2008, por primera vez en la historia se registra mayor número de matrícula particular subvencionada por sobre la matrícula municipal, existiendo un aumento de 2.901 escuelas particulares subvencionadas entre los años 1992 y 2010 (Fundación SOL, 2011). Si bien la competencia existe mayormente entre las escuelas urbanas, los datos de esta investigación muestran que en lo rural también existe competencia por la matrícula, aunque en menor proporción.

Sumado a lo anterior, el análisis que vincula el cierre con la vulnerabilidad de sus estudiantes, muestra que las escuelas cerradas estaban compuestas por estudiantes de los grupos más pobres del país. Esto es preocupante y amerita indagar en sus consecuencias tanto para los estudiantes y sus familias, como a nivel de políticas de desarrollo rural. Dentro de las regiones que concentran mayor cantidad de cierres en proporción a su número de escuelas es la región de La Araucanía, la cual se caracteriza por ser la de mayor pobreza (MINISTERIO DE DESARROLLO SOCIAL, 2018) y ser la segunda en concentrar mayor población perteneciente a pueblos originarios (INSTITUTO NACIONAL DE ESTADÍSTICAS, 2018).

Por otro lado, existe una tendencia en que la mayoría de las escuelas que cierran tienen una matrícula inferior a 12 estudiantes, las que son consideradas como pequeñas. Sin embargo, se evidencia el cierre de escuelas consideradas como grandes, lo cual nos permite comprender que éste no es el único criterio utilizado para cerrar un establecimiento.

\section{Discusiones}

Chile no cuenta con una adecuada definición de lo rural ni comprensión de sus ruralidades, lo que ha debilitado la construcción de políticas que vayan más allá de una visión economicista y que consideren la educación y lo social como ejes igualmente importantes. Una manifestación de ello es el cierre masivo de escuelas, que obedecen a estrategias verticalistas que no consideran ni la participación de la escuela, ni las necesidades comunitarias. Éste tipo de prácticas acarrea enormes efectos en un territorio, no sólo por desconocer las necesidades situadas de los ciudadanos, sino también los derechos de la ciudadanía a la participación y de los estudiantes a una educación de calidad.

Los argumentos para el cierre son de carácter financiero, adhiriendo a una racionalidad económica que maximiza la relación costo - beneficio; desconociendo la importancia de las variables sociales, comunitarias y de bienestar. Esto se acompaña de una visión funcionalista y tecnocrática de la escuela, que es concebida como una institución que entrega un servicio educativo dirigido a estudiantes; de este modo, cuando se cierran y se consolidan escuelas, la preocupación de las autoridades es mantener a los estudiantes dentro del sistema. Sin embargo, no se considera el rol que la escuela pública rural tiene en el desarrollo comunitario y en la construcción de identidad local, en particular en los contextos rurales latinoamericanos. Las escuelas 
rurales públicas están fuertemente orientadas al territorio y a las necesidades locales, lo cual se pone en evidencia durante situaciones de emergencia por ejemplo; frente a un desastre natural, la escuela es el centro neurálgico de la acción comunitaria (NÚÑEZ; SOTO, 2014). El cierre provoca un debilitamiento de la articulación y cohesión de la comunidad. Por nuestras investigaciones anteriores y otras internacionales, sabemos que el cierre de la escuela rural implica la desaparición del único articulador social que permanecía vigente en los pueblos, lo que afecta la cohesión social a distintos niveles, cercena sus posibilidades de desarrollo y sus proyecciones a futuro, puesto que trunca la llegada de nuevas familias con niños (NÚÑEZ; SOTO, 2014; SOLÍS; NÚÑEZ, 2014; KEARNS et al., 2009; AUTTI; HYRY-BEIHAMMER, 2014).

Uno de los desafíos pendientes para Chile, es la consolidación de una sociedad más participativa y democrática (SCHULZ et al., 2016). Esto cobra mayor relevancia aún en los contextos rurales (PROGRAMA PARA LAS NACIONES UNIDAS, 2008), donde primó el régimen de hacienda en los grandes latifundios hasta la primera mitad del siglo XX, asentándose fuertemente un patrón cultural que reproduce un estilo de autoridad hacendal hasta la actualidad (BENGOA, 2015; ARAUJO; BEYER 2013). Una mayor participación de los habitantes rurales en las decisiones que les afectan, implicaría el establecimiento de relaciones más democráticas por parte de las autoridades municipales. Desde esta perspectiva, el cierre no afecta solamente el derecho a la educación de los niños de un determinado territorio, sino también, el derecho a la participación de la comunidad local. Tal como el informe del PNUD (2008) sobre ruralidad en Chile sostiene, se requieren conversaciones que integren las perspectivas e intereses de los distintos actores que componen las sociedades rurales. En definitiva se necesita de otra institucionalidad para que exista mayor participación y democratización en lo rural. Un inicio podría ser abrir procesos de consulta comunitaria cuando se planifique el cierre de escuelas rurales.

Entender la escuela rural como un articulador de la identidad, bienestar y cohesión social, permite instalar la idea de la educación rural como un valor público (FELBER, 2012; LINEBAUGH, 2013). Esto implica considerar - en primer lugar- que una escuela no es pública porque es financiada por el Estado (RASCO, 2016), sino porque se identifica con la necesidades y valores de la ciudadanía (OSTROM, 2000). En segundo lugar, una escuela posee valor público en la medida en que atiende al bien común de una comunidad determinada (LAVAL, 2004; SANDEL, 2012) y en tercer lugar, agrega valor público en la medida en que cimenta valores pedagógicos como el laicismo, la inclusión, las virtudes públicas y el desarrollo de las capacidades en el alumnado (SEN, 2015; WINKLEY, 2011; MONGON; LEADBEATER, 2012). Podríamos sostener que las escuelas rurales han agregado efectivamente valor público a la educación, lo que es puesto en peligro por la racionalidad económica y el enfoque funcionalista; por lo tanto, su cierre debe ser evaluado a la luz del bien común que favorecen.

Por otro lado, a pesar que en Chile se crea en el año 2008 la Ley de Subvención Escolar Preferencial (CHILE, 2008), que constituye una política focalizada para aumentar la retención escolar de los estudiantes pobres, al mismo tiempo, es a esos estudiantes a quienes se les cierran las escuelas. No existe actualmente un catastro de las razones oficiales de cada cierre, por lo que se desconoce cuántos de estos obedecen a los bajos puntajes del SIMCE. Tal como señalamos, estos se correlacionan con el NSE de los estudiantes y el nivel de estudios de sus padres, por tanto, podría esperarse que los resultados de estas escuelas hayan sido bajos con relación al promedio nacional. Si el criterio del puntaje SIMCE para cerrar escuelas se aplicara tal como faculta la LGE, la población rural sería la más afectada. Este es otro antecedente que da cuenta de una falta de consideración de la población rural en el diseño de políticas.

Aun cuando frente al cierre se cautela la matrícula y los estudiantes son trasladados a otros establecimientos, lo cierto es que la movilidad estudiantil conlleva riesgos no considerados, tales 
como la disminución en el rendimiento escolar, la deserción (EADIE et al., 2013; LLERAS; MCKILLIP, 2016) e incluso, problemas de salud mental asociados a dificultades de integración social en la nueva escuela (SINGH et al., 2014). Resulta llamativo que los cierres hayan sido promovidos sin profundizar en las implicancias psicosociales que genera para los sujetos y las comunidades. Al igual que otros investigadores denuncian para el caso de China (MEI; FANG; YUANYAN, 2013), que después de años de cierre sistemático de escuelas rurales, no han existido estudios que evalúen si esta medida ha traído o no beneficios para el aprendizaje o la socialización de los estudiantes; tampoco existen en Chile estudios de esta índole que permitan evaluar los beneficios de los cierres.

Es posible que las consecuencias individuales y comunitarias sean aún más gravitantes para las escuelas que recibían a familias de pueblos originarios y que eran adecuadas culturalmente al contexto, puesto que su adaptación a nuevos establecimientos puede ser más difícil. Conviene destacar que la región de La Araucanía, que concentra la mayor cantidad de cierres, es una zona de conflicto entre el Estado y el Pueblo Mapuche (PADILLA, 2015), por lo que cerrar de manera sistemática la única institución de representación estatal que va quedando operativa en muchos de los poblados, es una decisión que puede terminar por debilitar aún más la cohesión social.

En el caso de los peak de cierre (2005 y 2010), es importante indagar en los elementos sociopolíticos que pueden vincularse a ellos. El año 2005 está dentro de un periodo nacional de mejoramiento de la economía, pero de ahorro según los lineamientos del gobierno de la época (presidente Ricardo Lagos, 2000 - 2006). Sin embargo, sería necesario hacer una indagación más profunda.

En el 2010, los resultados confirman lo planteado en nuestras investigaciones etnográficas, acerca de la relación de los cierres con el abordaje de las consecuencias del terremoto del 27 de febrero de dicho año. De un total de 8.326 establecimientos ubicados en la zona de desastre, entre Valparaíso y la Araucanía, 2.945 de dependencia municipal sufrieron algún daño (MINISTERIO SECRETARÍA GENERAL DE LA PRESIDENCIA, 2010), ante lo cual el Gobierno implementó un plan de reconstrucción. Emergió entonces la postura de investigadores que consideraron el desastre como una "oportunidad" para realizar mejoras, cerrando escuelas con bajos logros, tal como se hizo en el plan de reconstrucción de New Orleáns luego del huracán Katrina en USA; a partir del cual muchas escuelas fueron cerradas y reemplazadas por escuelas charter (ELACQUA; SANTOS; SALAZAR, 2010). Es así como el MINEDUC realizó un llamado a los alcaldes para promover la fusión de escuelas en las zonas devastadas, especialmente las rurales.

Entonces, si bien el cierre de escuelas rurales no ha sido declarado en Chile como una política oficial, como sí se ha hecho en otros países, en la práctica opera como tal. En ciertos momentos, como los de catástrofe, el Estado ha tenido un rol activo promoviendo los cierres y, en otros periodos más prolongados, ha sido a lo menos negligente, al no desarrollar mecanismos para evitar que los cierres se transformaran en una tendencia masiva, tal como los datos presentados muestran.

En cuanto al cambio de denominación, conviene indagar en sus consecuencias económicas y pedagógicas para las escuelas. Al perderse el piso rural, la escuela puede encontrarse en la antesala del cierre. Se requiere revisar la categorización dicotómica rural/urbana para la denominación de las escuelas, de modo de crear definiciones que se adapten a las transformaciones del contexto rural, tal como algunos investigadores proponen (RODRÍGUEZ et al., 2016). El marco de la "nueva ruralidad" puede ser de gran aporte para comprender la ruralidad actual y su dinamismo (RODRÍGUEZ; SALAS, 2010; KAY, 2009), puesto que los cambios en los planes reguladores de las localidades no debieran ser suficientes para redefinir un territorio como urbano. Hoy las localidades rurales pueden 
contar con calles pavimentadas o dedicarse al turismo, pero no por eso dejarán su condición rural automáticamente, dado que existen aspectos culturales que la política educativa no considera. Al respecto, existen propuestas conceptuales que pueden ser útiles, en tanto permiten repensar la relación entre las entidades rural/urbana como un continuo, lo que hace emerger las zonas intermedias, así como también la ruralidad comprendida desde gradientes, distinguiendo distintos niveles de ruralidad (OSSES; FOSTER; NÚÑEZ, 2006) al basarse en las categorías utilizadas por la OCDE (2014), que consideran niveles intermedios entre las zonas que se definen como predominantemente rurales o predominantemente urbanas.

\section{Notas}

1. Realizado en el marco del proyecto FONDECYT No 1161338.

2. Instrumento de planificación que define anualmente los planes de acción de la política de educación municipal.

3. Este dato se encontraba disponible en el sitio web del Ministerio de Educación hasta hace 2 años; sin embargo, ya no se existe en la plataforma.

4. SIMCE (Sistema de Medición de la Calidad de la Educación) fue creado el año 1988, siendo una evaluación externa al sistema educativo, de carácter estandarizado. Evalúa los resultados de aprendizaje de los establecimientos, en diferentes asignaturas o áreas de aprendizaje, por medio de una medición que se aplica a todos los estudiantes del país que cursan los niveles evaluados. Ver https://www.agenciaeducacion.cl/evaluaciones/que-es-el-simce/.

5. El año 2013 nace la campaña "Alto al Simce": Ver http://www.altoalsimce.org/. El año 2014 la revista Docencia editada por el Colegio de Profesores de Chile, publica un número especial sobre la temática SIMCE. Ver Revista Docencia (2014) SIMCE: Trastocando los sentidos de la educación pública. N 52, año XIX. En http://www.revistadocencia.cl/.

6. En el año 2007 se realiza una nueva división administrativa del territorio pasando de 13 a 15 regiones, por ello para el análisis regional se debió concentrar las regiones I - XIV y X - XV con el fin de volver a la antigua división de 13 regiones para así poder comparar los datos.

7. Este constructo se explica más adelante.

8. Las bases de datos utilizadas tienen carácter censal.

9. Corresponde al número de ubicación dentro del directorio oficial del MINEDUC, que se transforma en un número único e irreemplazable de identificación (e: ID).

10. Se considera este periodo de tiempo debido a que las bases de datos de IVE-SINAE se encuentran disponibles a partir del año 2010 .

\section{Contribución de los autores}

Cuestionamiento y conceptualización; Muñozo CN. Investigación; Muñozo CN, Niculcar BG, Grech S. Escritura; Muñozo CN, Niculcar BG, Grech S, Costa PA. Analizando; Niculcar BG.

\section{Referencias}

AGENCIA DE CALIDAD DE LA EDUCACIÓN. Evolución de las brechas socioeconómicas de rendimiento en pruebas SIMCE. Chile: Agencia de Calidad de la Educación, 2015.

ALBARRÁN, A. S. Sociología rural y nueva ruralidad sur-sur. Espacio Abierto, Venezuela, v. 25 n. 3, p. 49-63, 2016. Disponible en: https://produccioncientificaluz.org/index.php/espacio/article/view/22107. Acceso en: 12 de jul. de 2019.

ARAUJO, K.; BEYER, N. Autoridad y autoritarismo en Chile. Reflexiones en torno al ideal-tipo portaliano. Atenea, Concepción, n. 508, p. 171-185, 2013. https://doi.org/10.4067/S0718-04622013000200012.

ARVESETH, L. G. Friedman's School Choice Theory: The Chilean Education System. All Graduate 
Plan B and other Reports, Logan, n. 386, p. 1-28, 2014. Disponible en: https://digitalcommons.usu. edu/cgi/viewcontent.cgi? article=1378\&context=gradreports. Acceso en: 20 may. 2018.

AUTTI, O.; HYRY-BEIHAMMER, E. K. School closures in rural Finnish communities. Journal of Research in Rural Education, Pensilvânia, v. 29, n. 1, p. 1-17, 2014. Disponible en: http://jrre.vmhost. psu.edu/wp-content/uploads/2014/03/29-1.pdf. Acceso en: 10 jun. 2018.

BENGOA, J. Historia rural de Chile central. Tomo II, Crisis y ruptura del poder hacendal. Santiago: LOM, 2015.

BORÓN, A. State, capitalism and democracy in Latinamerica. Colección Secretaria Ejecutiva. Ciudad de Buenos Aires: Consejo Latino Americano de Ciencias Sociales, 2003.

CHILE. Ley Número 20.248, de 25 de enero de 2008. Establece Régimen de Subvención Escolar Preferencial. Ministerio de Educación: Santiago, 2008. Disponible en: https://www.leychile.cl/ Navegar?idNorma=269001. Acceso en: 11 de nov. de 2018.

CHILE. Ley Número 20.370, de 17 de Agosto de 2009. Establece la Ley General De Educación. Ministerio de Educación: Santiago, 2009. Disponible en: https://www.leychile.cl/Navegar?idNorma=1006043. Acceso en: 11 de nov. de 2018.

DARVILLE, P.; RODRÍGUEZ, J. Institucionalidad, financiamiento y rendición de cuentas en educación. Santiago: Estudios de Finanzas Públicas, Gobierno de Chile, 2007. Disponible en: https:// www.dipres.gob.cl/598/articles-21658_doc_pdf.pdf. Acceso en: 3 de mar. de 2020

DATTWYLER, R. A. R.; ALVARADO, V.; ARENAS, F.; SALAZAR, A.; VOLKER, P. La comunidad disidente: reacción, métodos y conflictividad socio-espacial en el borde costero de la región de Valparaíso. ACE: Arquitectura, Ciudad y Entorno, Catalunya, v. 10, n. 30, p. 31-56, 2016. https:// doi.org/10.5821/ace.10.30.3867.

EADIE, S.; EISNER, R.; MILLER, B.; WOLF, L. Student Mobility Patterns and Achievement in Wisconsin. Universtity of Wisconsin - Madison, Workshop in Public Affairs, 2013. Disponible en: http://www.lafollette. wisc.edu/images/publications/workshops/2013-DPI_mobility.pdf. Acceso en: 20 ago. 2018.

ELACQUA, G; SANTOS, H.; SALAZAR, F. Terremoto 27/2: Oportunidad para mejorar las escuelas en Chile. Santiago: Centro de Políticas Comparadas de Educación, Universidad Diego Portales, 2010.

FELBER, C. La economía del bien común. Barcelo: Deusto S.A. Ediciones, 2012.

FUNDACIÓN SOL. El desalojo de la educación pública. Santiago: Fundación SOL, 2011. Disponible en: http://www.fundacionsol.cl/estudios/el-desalojo-de-la-educacion-publica/. Acceso en: 6 jun. 2019.

GOBIERNO DE CHILE. Plan Araucanía: Invirtiendo en Personas y Oportunidades. Santiago: Gobierno de Chile, 2010. Disponible en: https://issuu.com/planaraucania/docs/plan_araucania_7. Acceso en: 20 mar. 2018.

INSTITUTO NACIONAL DE ESTADÍSTICAS. Síntesis de resultados censo 2017. Santiago: Instituto Nacional de Estadísticas, 2018. Disponible en: https://www.censo2017.cl/descargas/home/sintesis-deresultados-censo2017.pdf. Acceso en: 18 jul. 2018. 
INSTITUTO NACIONAL DE ESTADÍSTICAS. Síntesis de resultados Censo Nacional 2002. Santiago: Instituto Nacional de Estadísticas, 2013. Disponible en: https://www.ine.cl/docs/default-source/censode-poblacion-y-vivienda/publicaciones-y-anuarios/2002/sintesiscensal-2002.pdf. Acceso en: 18 jul. 2018.

JIMÉNEZ, V.; HIDALGO, R.; CAMPESINO, A.-J.; ALVARADO, V. Normalización del modelo neoliberal de expansión residencial más allá del límite urbano en Chile y España. Revista EURE Revista de Estudios Urbano Regionales, Santiago, v. 44, n. 132, p. 27-46, 2018. https://doi.org/10.4067/ s0250-71612018000200027.

KAY, C. Estudios rurales en América Latina en el periodo de globalización neoliberal: ¿una nueva ruralidad?. Revista Mexicana de Sociología, México, v. 71, n. 4, p. 607-645, 2009.Disponible en: http://www.scielo.org.mx/scielo.php?script=sci_arttext\&pid=S0188-25032009000400001. Acceso en: 8 de nov. de 2018.

KEARNS, R. A.; LEWIS, N.; MCCREANOR, T.; WITTEN, K. 'The status quo is not an option': Community impacts of school closure in South Taranaki, New Zealand. Journal of Rural Studies, Reino Unido, v. 25, n. 1, p. 131-140, 2009. https://doi.org/10.1016/j.jrurstud.2008.08.002.

LAVAL, L. La escuela no es una empresa: el ataque neoliberal a la enseñanza pública. Buenos Aires: Paidós, 2004.

LINEBAUGH, P. El manifiesto de la carta magna - comunes y libertades para el pueblo. Madrid: Traficantes de Sueños, 2013.

LLERAS, C. H.; MCKILLIP, M. When children move: Behavior and achievement outcomes during elementary school. The Journal of Educational Research, Filadelfia, v. 110, n. 2, p. 177-187, 2017. https://doi.org/10.1080/00220671.2015.1060930.

MARCEL, M.; TOKMAN, C. ¿Cómo se financia la educación en Chile?. Estudios de Finanzas Públicas. Chile: Gobierno de Chile, Ministerio de Hacienda, 2005. Disponible en: https://www.dipres. gob.cl/598/articles-21669_doc_pdf.pdf.. Acceso en: 11 de nov. de 2018.

MARTINIS, P.; REDONDO, P. (comps.). Igualdad y educación. Escrituras entre dos orillas. Buenos Aires: Del Estante Editorial, 2006.

MEI, D.; FANG, C.; YUANYAN, B. An Empirical Study on the Effect of School Consolidation in Rural Areas on Student Achievement: A Case Study of Shaanxi Province. Chinese Education \& Society, Filadelfia, v. 46, n. 5, p. 56-70, 2013. https://doi.org/10.2753/CED1061-1932460504.

MINISTERIO DE DESARROLLO SOCIAL. Pobreza y distribución de ingresos. Presentación de resultados Casen 2017. Observatorio Social. 2018. Disponible en: http://observatorio.ministeriodesarrollosocial.gob. cl/casen-multidimensional/casen/docs/Resultados_pobreza_Casen_2017.pdf. Acceso en: 3 de mar. de 2020.

MINISTERIO SECRETARÍA GENERAL DE LA PRESIDENCIA, DIVISIÓN JURÍDICO-LEGISLATIVA. Plan de Reconstrucción Terremoto y Maremoto del 27 de febrero de 2010. Santiago: Gobierno de Chile, 2010. Disponible en: https://www.preventionweb.net/files/28726_plandereconstruccinagosto2010.pdf. Acceso en: 3 de mar. de 2020

(MINEDUC) MINISTERIO DE EDUCACIÓN. Fija texto refundido, coordinado y sistematizado del decreto con fuerza de ley no 2 , de 1996 , sobre subvención del estado a establecimientos 
educacionales. Santiago: Ministerio de Educación, 1998. Disponible en: https://www.leychile.cl/ Navegar?idNorma=127911\#rural0. Acceso en: 20 ago. 2018.

MONGON, D.; LEADBEATER, C. Measuring for value. In School Leadership for Public Value. Understanding valuable outcomes for children, families and communities, 1st ed., p. 86-101, 2012. Institute of Education Publications.

NÚÑEZ, C. G.; SOTO, S. C. R. ¿Qué sucede en las comunidades cuando se cierra la escuela rural? Un análisis psicosocial de la política de cierre de las escuelas rurales en Chile. Universitas Psychologica, 13(2), 615-625, 2014.

OCDE. OECD Rural Policy Reviews. Chile: OECD Publishing, 2014.

OLIVARES, P. A.; TOMÁS, R. B.; JIMÉNEZ, A. B.; PEÑAFIEL, L. D.; CEBRIÁN, V. D.; GARZARÁN, R. M. R. La escuela rural y la política educativa española. Diferencias entre Comunidades Autónomas: Andalucía, Aragón y Cataluña. International Studies on Law and Education, São Paulo, 2015. Disponible en: https://zaguan.unizar.es/record/56204/files/texto_completo.pdf. Acceso en: 22 ago. 2018.

OSSES, P.; FOSTER, W.; NÚÑEZ, R. Medición de niveles de ruralidad y su relación con actividades económicas en la X Región de los Lagos - Chile. Enfoque Geográfico - Económico. Research in Agricultural \& Applied Economics, Kentucky, v. 10, n. 12, p. 107-118, 2006. Disponible en: https:// ageconsearch.umn.edu/bitstream/97361/2/Osses \%20et \%20al.pdf. Acceso en: 12 jul. 2018.

OSTROM, E. El gobierno de los bienes comunes: la evolución de las instituciones de acción colectiva. México: Fondo de cultura económica, 2000.

PADILLA, F. P. Malón. La rebelión del movimiento Mapuche 1990-2013. Revista de Historia Social y de las Mentalidades, Santiago, v. 19, n. 1, p. 181-187, 2015. Disponible en: http://www.revistas.usach. cl/ojs/index.php/historiasocial/article/view/2134. Acceso en: 12 jul. 2018.

PÉREZ, C. La emancipación de la escuela rural aún no ha llegado: historia de la educación primaria rural en Chile (1920-1970). Chile: Museo de la Educación, 2018. Disponible en: https:// www.museodelaeducacion.gob.cl/648/articles-87328_archivo_PDF.pdf. Acceso en: 12 jul. 2018.

PROGRAMA DE LAS NACIONES UNIDAS PARA EL DESARROLLO (PNUD). Desarrollo Humano en Chile Rural. Seis Millones por Nuevos Caminos. Chile: Programa de las Naciones Unidas para el Desarrollo, 2008.

RASCO, J. F. A. Lo público y lo privado en la escuela pública. Revista Interuniversitaria de Formación del Profesorado, Murcia, v. 30, n. 1, p. 17-24, 2016. Disponible en: https://recyt.fecyt.es/index.php/ RIFOP/issue/view/2863/showToc. Acceso en: 12 jul. 2018.

RODRÍGUEZ, J. C.; SALAS, H. La Ruralidad Chilena Actual. Aproximaciones desde la Antropología. En: HERNÁNDEZ, Roberto; PEZO, Luis (ed.). La ruralidad chilena actual, Chile: CoLibris, 2010. p. $45-78$.

RODRÍGUEZ, L. G. La primaria durante la dictadura: reforma curricular y “concentración” de escuelas rurales. Archivos de Ciencias de la Educación (4a. época), Argentina, n. 2, p. 85-102, 2008. Disponible en:http://www.memoria.fahce.unlp.edu.ar/art_revistas/pr.3177/pr.3177.pdf. Acceso en: 10 jun. 2018. 
RODRÍGUEZ, P.; TRUFFELLO, R.; SUCHANB, K.; VARELA, F.; MATASA, M.; MONDACA, J.; CÉSPEDES, D.; VALENZUELA, L.; VALENZUELA, J. P.; ALLENDE, C. Apoyando la formulación de políticas públicas y toma de decisiones en educación utilizando técnicas de análisis de datos masivos: el caso de Chile. Technical Report, Chile, p. 1-12, 2016. https://doi.org/10.13140/RG.2.1.1419.9283.

RODRÍGUEZ, S. G. Ciudadanía rural y nueva ruralidad: impacto de las Modernizaciones en el medio rural de Chile. Contextos: Estudios de Humanidades y Ciencias Sociales, Chile, n. 32, p. 71-78, 2014. Disponible en: http://revistas.umce.cl/index.php/contextos/article/view/276. Acceso en: 5 de ene. 2019.

ROJAS, H. L. Nueva ruralidad, psicología y educación. Revista Athenea, Barcelona, n. 8, p. 36-45, 2017.

SANDEL, M. What money can't buy? The moral limits of markets. London: Allen Lane, 2012.

SCHULZ, W.; AINLEY, J.; COX, C.; FRIEDMAN, T. Estudio internacional sobre educación cívica y ciudadana 2016 de la IEA. Percepciones de los jóvenes acerca del gobierno, la convivencia pacífica y la diversidad en cinco países de América Latina. Ámsterdam: IEA, 2016. Disponible en: https:// www.iea.nl/sites/default/files/2019-05/ICCS\%202016\%20Latin\%20American\%20Report\%20Spanish. pdf. Acceso en: 12 jul. 2018.

SEN, A. The idea of justice: A response, Philosophy \& Social Criticism, v. 41, n. 1, p. 77-88, 2015. https://doi.org/10.1177/0191453714553501.

SINGH, S. P.; WINSPER, C.; WOLKE, D.; BRYSON, A. School mobility and prospective pathways to psychotic-like symptoms in early adolescence: a prospective birth cohort study. Journal of the American Academy of Child \& Adolescent Psychiatry, Nueva York, v. 53, n. 5, p. 518-527, 2014. https://doi.org/10.1016/j.jaac.2014.01.016.

SOLÍS-ARAYA, C.; NÚÑEZ-MUÑOZ, C. El cierre de la escuela rural y la integración social de familias en el contexto educativo urbano: un estudio de caso en la zona sur de Chile. Revista Iberoamericana de Evaluación Educativa. [S.l.], v. 7, n. 3, 2014. Disponible en: https://revistas.uam.es/index.php/ riee/article/view/3102. Acceso en: 03 mar. 2020.

TRIMANO, L. Integración social y nueva ruralidad: ser ¿"hippie”? en el campo. Revista de Antropología Social, Madrid, v. 24, p. 317-348, 2015. https://doi.org/10.5209/rev_RASO.2015.v24.50660.

WINKLEY, D. Public Value in Education: A case-Study. In J. Benington \& M. H. Moore (Eds.), Public Value. Theory and Practice p. 244-254, 2011. New York: Palgrave Macmillan.

\section{Sobre los autores}

Carmen Núñez Muñoz, Profesora Adjunta de la Escuela de Psicología de la Pontificia Universidad Católica de Valparaíso (PUCV), es Doctora en Ciencias de la Educación por la Pontificia Universidad Católica de Chile en cotutela con la Université Bordeaux 2 Victor Segalen, Psicóloga y Licenciada en Psicología de la Pontificia Universidad Católica de Valparaíso. Su línea de investigación es la 
educación rural, siendo Investigadora Responsable de proyectos financiados por la Comisión Nacional de Investigación Científica y Tecnológica CONICYT. Uno de sus principales focos de estudio ha sido los efectos del cierre de escuelas rurales en Chile.

Bryan González Niculcar, Psicólogo y Licenciado en Psicología de la Pontificia Universidad Católica de Valparaíso, profesor agregado de la escuela de Psicología de la Pontificia Universidad Católica de Valparaíso. Cuenta con experiencia de trabajo en diversos proyectos de investigación e innovación en temáticas vinculadas a la educación y las transformaciones de los espacios rurales.

Paula Ascorra Costa, Profesora Titular de la Escuela de Psicología de la Pontificia Universidad Católica de Valparaíso (PUCV). Doctora en Psicología de la Universidad de Chile. Investigadora Principal del Centro de Investigación para la Educación Inclusiva. Directora de la Escuela de Psicología de la PUCV. Su línea de investigación es gestión de la convivencia escolar y el manejo de estados de ánimo en contextos organizacionales. Investigadora Responsable de diversos proyectos financiados por la Comisión Nacional de Investigación Científica y Tecnológica de Chile, CONICYT y del Fondo de Investigación del Ministerio de Educación de Chile. Es autora de diversos artículos, capítulos de libro y libros.

Shaun Grech, Director de The Critical Institute, Malta y Editor de la revista internacional Disability and the Global South. Doctor en Estudios de la Discapacidad por la Manchester Metropolitan University en Inglaterra. Las áreas clave de investigación incluyen discapacidad, ruralidad y pobreza extrema con un enfoque especial en América Latina. Es autor y editor de diversos libros y ha publicado extensamente en revistas internacionales establecidas.

Recibido: 30 Oct 2018

Acepto: 14 Jan 2019 